\title{
Effect of fresh frozen plasma on the in vitro activation of U937 monocytes: a potential role for the age of blood donors and their underlying cytokine profile
}

\author{
Mariana Patlán 1,2, Fausto Sánchez-Muñoz², Luis M. Amezcua-Guerra²,3, Adriana Granados², Araceli Páez ${ }^{4}$, \\ Felipe Massó ${ }^{4}$, Ana M. Mejía ${ }^{5}$, Angeles Soster ${ }^{5}$, Rafael Bojalili2, ${ }^{2,}$ Lenin Pavón ${ }^{6}$, Luis A. Jiménez-Zamudio ${ }^{*^{*}}$ \\ and Ricardo Márquez-Velasco ${ }^{2^{*}}$ (D)
}

\begin{abstract}
Background: Fresh frozen plasma (FFP) administration may increase the risk of nosocomial infections in parallel with the development of immune modulation. This could be driven by soluble mediators, possibly influencing the in vitro activation of human U937 monocyte cells, in a manner dependent on the age of the donors.

Methods: FFP donors were stratified into groups of 19-30 years, 31-40 years or 41-50 years, and U937 cells were cultured with FFP (alone or plus lipopolysaccharide-LPS) for $24 \mathrm{~h}$. Both in FFP and supernatants, TNF, IL-1 $\beta$, IL-6, and IL-10 levels were measured by ELISA. Additionally, CD11B, TLR2, and CASP3 gene expression were measured by qtPCR in U937 cells. Total phagocytic activity was also assayed.

Results: Elevated IL-10, but low TNF and IL-1 $\beta$ levels were measured in FFP from individuals aged 19-40 years, whereas in individuals aged 41-50 years FFP were characterized by equalized TNF and IL-10 levels. Elevated IL-6 levels were found in all FFP samples, especially in those from the oldest individuals. FFP stimulation was associated with striking modifications in cytokine production in an age-dependent way. Exposure to FFP attenuates the response to LPS. TLR2 and CD11B expression were enhanced regardless of the age of plasma donors, although CASP3 expression was increased only when FFP from individuals aged 19-40 years were tested. Phagocytosis decreased after exposure to FFP regardless of donor age.
\end{abstract}

Conclusion: Our results suggest that soluble mediators in FFP may modulate the functioning of monocytes. Interestingly, this effect appears to be partially influenced by the age of donors.

Keywords: Fresh frozen plasma, Monocytes, Cytokines, Inflammation

\section{Background}

Fresh frozen plasma (FFP) is the fluid portion obtained from one unit of blood, which is processed by

\footnotetext{
*Correspondence: lajimenezz@gmail.com; marquezric@hotmail.com 2 Department of Immunology, Instituto Nacional de Cardiología Ignacio Chávez, Juan Badiano No1, Col Sección XVI, 14080 Mexico City, Mexico ${ }^{7}$ Department of Immunology, Escuela Nacional de Ciencias Biológicas, Instituto Politecnico Nacional, Prolongación de Carpio y Plan de Ayala s/n, Santo Tomas, 11340 Mexico City, Mexico

Full list of author information is available at the end of the article
}

centrifugation and frozen at $-18{ }^{\circ} \mathrm{C}$ until used. It contains many soluble molecules such as clotting factors, fibrinogen, albumin, electrolytes, and natural anticoagulants $[1,2]$. The use of FFP as adjunct therapy is common in different clinical settings. For instance, more than 4,000,000 units of FFP are transfused in the United States each year [3], and approximately $30 \%$ of patients in intensive care units will receive FFP transfusion at any point during their hospitalization $[4,5]$. However, transfusion of FFP and other hemoderivatives has also 
been associated with abnormalities in the inflammatory response [6-8], thus increasing the risk of infections and other adverse outcomes $[9,10]$. A few in vitro models such as co-cultures of monocytes with packed red blood cells [11] and co-incubation with FFP plus lipopolysaccharide (LPS) have been consistent to demonstrate immune disturbances characterized by decreased release of tumor necrosis factor (TNF) in parallel with increased interleukin (IL)-10 levels [12]. Although the underlying mechanisms are poorly understood, it is possible that soluble mediators such as cytokines in blood derivatives may modulate the functioning of monocytes and other leukocytes [13-17]. In support, development of post-transfusion side effects, including anaphylaxis, hemolytic reactions, and transfusion-related acute lung injury (TRALI) has been associated with the presence of cytokines in FFP and other blood derivatives [18-21]. Furthermore, these mediators are typically found in sera from healthy individuals and these may be also identified in leukocyte-reduced blood derivatives including FFP $[14,22,23]$.

In the notion that both type and level of circulating cytokines may vary according to age [24-26], we sought to explore the effect of FFP from age-stratified healthy blood donors on the in vitro activation of human cells from monocytic lineage.

\section{Methods}

\section{Plasma samples}

FFP from healthy donors were obtained by single-step heavy spin centrifugation ( $5000 \mathrm{~g}$ for 5-7 $\mathrm{min}$ ) in the blood bank at the Instituto Nacional de Cardiología Ignacio Chávez in Mexico City, Mexico [27]. Only FFP samples from male individuals aged 18-50 years old, weighing $>50 \mathrm{~kg}$, and fasting for more than $6 \mathrm{~h}$ were included. Blood samples fulfilled all the scrutiny tests required by the local regulation [28]. Accordingly, individuals with hepatitis $B$ or $C$ viruses, human immunodeficiency virus, herpes, pneumonia, diabetes, coronary artery disease, neoplasm, blood diseases, diarrhea or acute respiratory disease, syphilis, gonorrhea, malaria, drugs use, and users of acupuncture or with a history of tattoos are excluded as potential donors.

Ten $\mathrm{mL}$ of FFP were obtained during the routinely collection and processing in the blood bank. Samples were processed in free-pathogen conditions and stored in aliquots at $-70{ }^{\circ} \mathrm{C}$ until used. For analysis, FFP samples were grouped according to the age of donors as follows: $19-30$ years old $(n=11), 31-40$ years old $(n=9)$, and $41-50$ years old $(n=4)$.

The protocol was approved by the local ethics committee and study was performed in line with the Declaration of Helsinki and local regulations.

\section{Biochemical analyses}

TNF, IL-1 $\beta$, IL- 6 and IL-10 levels were measured in FFP samples and supernatant of cultured cells by enzymelinked immunosorbent assay (ELISA) using commercial kits according to the manufacturer's instructions (BioLegend, San Diego, CA, USA).

\section{Cell cultures}

The human monocyte U937 cell line was obtained from ATCC (Cat. \#CRL-1593.2) and cultured in RPMI-1640 with sodium bicarbonate and HEPES (Sigma, Palo Alto, CA, USA), supplemented with $1 \%$ L-glutamine (Sigma), $10 \%$ heat-inactivated fetal bovine serum (Gibco-Invitrogen, CA, USA), and 1\% streptomycin-penicillin (GibcoInvitrogen). A total of $7.5 \times 10^{5} \mathrm{U} 937$ cells in a final volume of $2 \mathrm{~mL} /$ well containing $1000 \mathrm{IU}$ of heparin/well as anticoagulant were placed in 24-well plates (Nunc, Denmark), incubated at $37{ }^{\circ} \mathrm{C}, 5 \% \mathrm{CO}_{2}$, and cultured as follows: (1) RPMI medium only (negative control); (2) RPMI medium plus $1 \mu \mathrm{g} / \mathrm{mL}$ of $E$. coli serotype 055 : B5 LPS (positive control); (3) RPMI medium plus 20\% FFP; (4) RPMI medium plus 40\% FFP; (5) RPMI medium with $20 \%$ FFP plus LPS; and (6) RPMI medium with $40 \%$ FFP plus LPS. Cultures were incubated for $24 \mathrm{~h}$, supernatants were collected, centrifuged $\left(3000 \mathrm{rpm}\right.$ at $\left.4{ }^{\circ} \mathrm{C}\right)$, and stored in $1.5 \mathrm{~mL}$ aliquots at $-75^{\circ} \mathrm{C}$. Of note, each FFP was tested individually.

In addition, U937 cells were placed in vials with $750 \mu \mathrm{L}$ Tripure Reagent (Roche Cat. 11667165001, Mannheim Germany) and frozen at $-75^{\circ} \mathrm{C}$ until studied.

\section{Reverse transcription and polymerase chain reaction}

To assess the expression of activation-related gene transcripts, RNA was extracted from U937 cells according to the manufacturer's recommendations. Electrophoresis in $1 \%$ agarose gel was used to assess RNA integrity, and concentration and purity by absorbance $268 / 280 \mathrm{~nm}$ in a Nanodrop 100 (Thermo, USA). cDNA was generated using $250 \mathrm{ng}$ of total RNA, random primers, and the Transcriptor first strand cDNA synthesis kit (Roche Cat. 04379012001, Mannheim Germany). Real time PCR was conducted with LNA hydrolysis probes from the Universal Probe Library Roche (UPL) (Roche, Cat 04683633001, Mannheim Germany), and intron spanning primers from Sigma. TLR2 (NM_003264.3) F 5'-CGTTCTCTCA GGTGACTGCTC-3', R'3-TCTCCTTTGGATCCTGCT TG-'5, UPL probe 14; CASP3 (NM_004346.3) F 5'-CTGGT TTTCGGTGGGTGT-3', R 3'-CCACTGAGTTTTCAGT GTTCTCC-5' UPL probe 34; CD11B (NM_000632.3) F 5'-GGCATCCGCAAAGTGGTA-3', R 3'-GGATCTTAA AGGCATTCTTTCG-5' UPL probe 9; and GADPH (NM_002046.3) F 5'-AGCCACATCGCTCAGACAC-3', R 3'-GCCCAATACGACCAAATCC-5' UPL probe 60. 
One $\mu \mathrm{L}$ of each cDNA was amplified with $400 \mathrm{nM}$ of primers, $100 \mathrm{nM}$ of UPL probe, with the LightCycler TaqMan ${ }^{\circledR}$ Master (Roche, Cat. 04535286001) followed by 45 cycles of $95^{\circ} 10 \mathrm{~s}, 60^{\circ} 60 \mathrm{~s}$, and $72^{\circ} 1 \mathrm{~s}$. Reference gene GAPDH was used for relative quantification, according to the $2^{-\Delta \Delta \mathrm{Ct}}$ method, using the non-stimulated cells as calibrator sample.

\section{Phagocytosis assays}

To evaluate phagocytic activity, U937 cells $\left(5 \times 10^{5}\right.$ cells/ $\mathrm{mL}$, in 24-well plates) were differentiated to macrophages by incubating with $2 \mathrm{ng} / \mathrm{mL}$ phorbol myristate acetate (PMA Cat P8139, Sigma) during 48 h. A phagocytosis assay kit (Cayman Chemical, USA) was performed according to the manufacturer's instructions. Briefly, rabbit IgG FITC-latex beads solution was added in cell cultures $(100 \mu \mathrm{L} / \mathrm{mL})$, and these were incubated during $24 \mathrm{~h}$ at $37{ }^{\circ} \mathrm{C}, 5 \% \mathrm{CO}_{2}$. Phagocytosis of FITC-labeled latex beads was assessed using a FACSCalibur (BD Bioscience, San Diego, CA, USA) flow cytometer.

\section{Statistical analysis}

The results of cytokine levels and expression of molecules are expressed as mean \pm standard error of mean, while phagocytosis activity is presented as percentage. Comparisons between groups were computed by the KruskalWallis test (with multiple comparison post-test by Dunn). Analyses were 2 -tailed, and a $P<0.05$ value was set for significance.

The GraphPad Prism 4.02 software (GraphPad Inc, San Diego, CA, USA) was used for calculations.

\section{Results}

Main demographic characteristics of blood donors are summarized in Table 1. As noted, characteristics such as weight, height, heart rate, diastolic and systolic blood pressure, temperature and serum levels of C-reactive

Table 1 Main clinical characteristics of healthy blood donors

\begin{tabular}{|c|c|c|c|}
\hline & $\begin{array}{l}19-30 \text { years } \\
(n=11)\end{array}$ & $\begin{array}{l}31-40 \text { years } \\
(n=9)\end{array}$ & $\begin{array}{l}41-50 \text { years } \\
(n=4)\end{array}$ \\
\hline Weight, Kg & $75.3 \pm 4.1$ & $71.5 \pm 2.4$ & $80.9 \pm 9.5$ \\
\hline Height, cm & $164.3 \pm 2.4$ & $168.4 \pm 1.5$ & $167.0 \pm 2.1$ \\
\hline Heart rate, bpm & $69.5 \pm 2.2$ & $63.1 \pm 1.7$ & $68.5 \pm 2.0$ \\
\hline $\begin{array}{l}\text { Systolic blood pressure, } \\
\mathrm{mm} \mathrm{Hg}\end{array}$ & $132.2 \pm 3.8$ & $126.0 \pm 5.3$ & $117.3 \pm 4.6$ \\
\hline $\begin{array}{l}\text { Diastolic blood pressure, } \\
\mathrm{mm} \mathrm{Hg}\end{array}$ & $75.4 \pm 3.9$ & $71.1 \pm 3.4$ & $73.5 \pm 4.2$ \\
\hline C-reactive protein, mg/L & $1.1 \pm 0.4$ & $1.0 \pm 0.2$ & $2.3 \pm 1.2$ \\
\hline
\end{tabular}

All data are presented as mean \pm standard deviation protein were not significantly different among individuals when they were grouped by age.

\section{Cytokines in fresh frozen plasma}

Cytokine levels were measured in FFP, and different inflammatory profiles were observed according to the age group. In donors aged 31-40 years, TNF concentration was $31.0 \pm 17.3 \mathrm{pg} / \mathrm{mL}$, IL- $1 \beta$ was $5.5 \pm 2.0 \mathrm{pg} /$ $\mathrm{mL}$ and IL-10 was $445.5 \pm 413.2 \mathrm{pg} / \mathrm{mL}$, whereas these figures were $10.7 \pm 4.2,1.3 \pm 1.3$, and $9.0 \pm 5.2 \mathrm{pg} / \mathrm{mL}$, respectively, in donors aged $41-50$ years. Plasma of 19 to 30 -year-old donors contained $16.6 \pm 6.5 \mathrm{pg} / \mathrm{mL}$ of TNF, $2.2 \pm 0.9 \mathrm{pg} / \mathrm{mL}$ of IL-1 $\beta$, and $30.4 \pm 17.8 \mathrm{pg} / \mathrm{mL}$ of IL-10 (Fig. 1, panels a, b, d). High IL-6 levels were detected in all FFP $(19-30$ years $=727.0 \pm 222.0 \mathrm{pg} / \mathrm{mL}$, $31-40$ years $=768.0 \pm 519.0 \mathrm{pg} / \mathrm{mL}$, and $41-50$ years $=$ $1501.0 \pm 1071.0 \mathrm{pg} / \mathrm{mL}$ ) (Fig. 1, panel c).

\section{U937 cells stimulated with $\mathbf{2 0 \%}$ fresh frozen plasma}

TNF levels in the supernatant of unstimulated U937 cells were $28.2 \pm 1.9 \mathrm{pg} / \mathrm{mL}$, while the exposure to $20 \%$ FFP induced levels of $36.7 \pm 6.9,36.5 \pm 13.5$ and $63.1 \pm 38.4 \mathrm{pg} / \mathrm{mL}$ in $19-30$ years, $31-40$ years and 41-50 years groups, respectively (Fig. 2, panel a). Stimulation with LPS increased TNF production $(64.1 \pm 5.2 \mathrm{pg} / \mathrm{mL})$ while co-stimulation with $20 \%$ FFP plus LPS induced age-dependent increments (1930 years $=59.6 \pm 17.1,31-40$ years $=104.8 \pm 51.9$, $41-50$ years $=140.8 \pm 88.8 \mathrm{pg} / \mathrm{mL} ; \mathrm{P}=\mathrm{ns}$ ) (Fig. 2, panel b). In addition, $0.9 \pm 0.9 \mathrm{pg} / \mathrm{mL}$ of IL- $1 \beta$ were found in unstimulated U937 cells, and these levels increased after stimulation with $20 \%$ FFP $(19-30$ years $=21.9 \pm 9.3 \mathrm{pg} /$ $\mathrm{mL}, 31-40$ years $=16.0 \pm 4.5 \mathrm{pg} / \mathrm{mL}$ ) reaching the highest level with stimulation with FFP of 41-50 years $(45.0 \pm 30.9 \mathrm{pg} / \mathrm{mL} ; \mathrm{P}<0.01)$ (Fig. 2, panel c). LPS stimulation increased levels of IL-1 $\beta(14.0 \pm 0.6 \mathrm{pg} / \mathrm{mL})$, but unexpectedly, co-stimulation with $20 \%$ FFP plus LPS did not modify IL- $1 \beta$ levels compared to cells stimulated with FFP alone (Fig. 2, panel d).

In unstimulated cultures, IL-6 level was $18.4 \pm 1.8 \mathrm{pg} /$ $\mathrm{mL}$, and exposure to $20 \%$ FFP induced $27.9 \pm 13.2$, $7.8 \pm 6.5$ and $60.7 \pm 51.6 \mathrm{pg} / \mathrm{mL}$ as challenged with FFP of 19-30, 31-40, and 41-50 years, respectively (Fig. 2, panel e). LPS stimulation induced a significant increase in the IL-6 levels $(126.5 \pm 8.0 \mathrm{pg} / \mathrm{mL})$, while co-stimulation with FFP + LPS was associated with modifications in the IL-6 levels as follows: $19-30$ years $=29.1 \pm 12.5 \mathrm{pg} /$ $\mathrm{mL}, \quad 31-40$ years $=6.3 \pm 6.3 \mathrm{pg} / \mathrm{mL}$, $41-50$ years $=52.3 \pm 43.4 \mathrm{pg} / \mathrm{mL}(\mathrm{P}<0.001$ for all comparisons) (Fig. 2, panel f). Finally, $20.9 \pm 1.2 \mathrm{pg} /$ $\mathrm{mL}$ of IL-10 were detected in unstimulated cultures, and these were not significantly modified by 

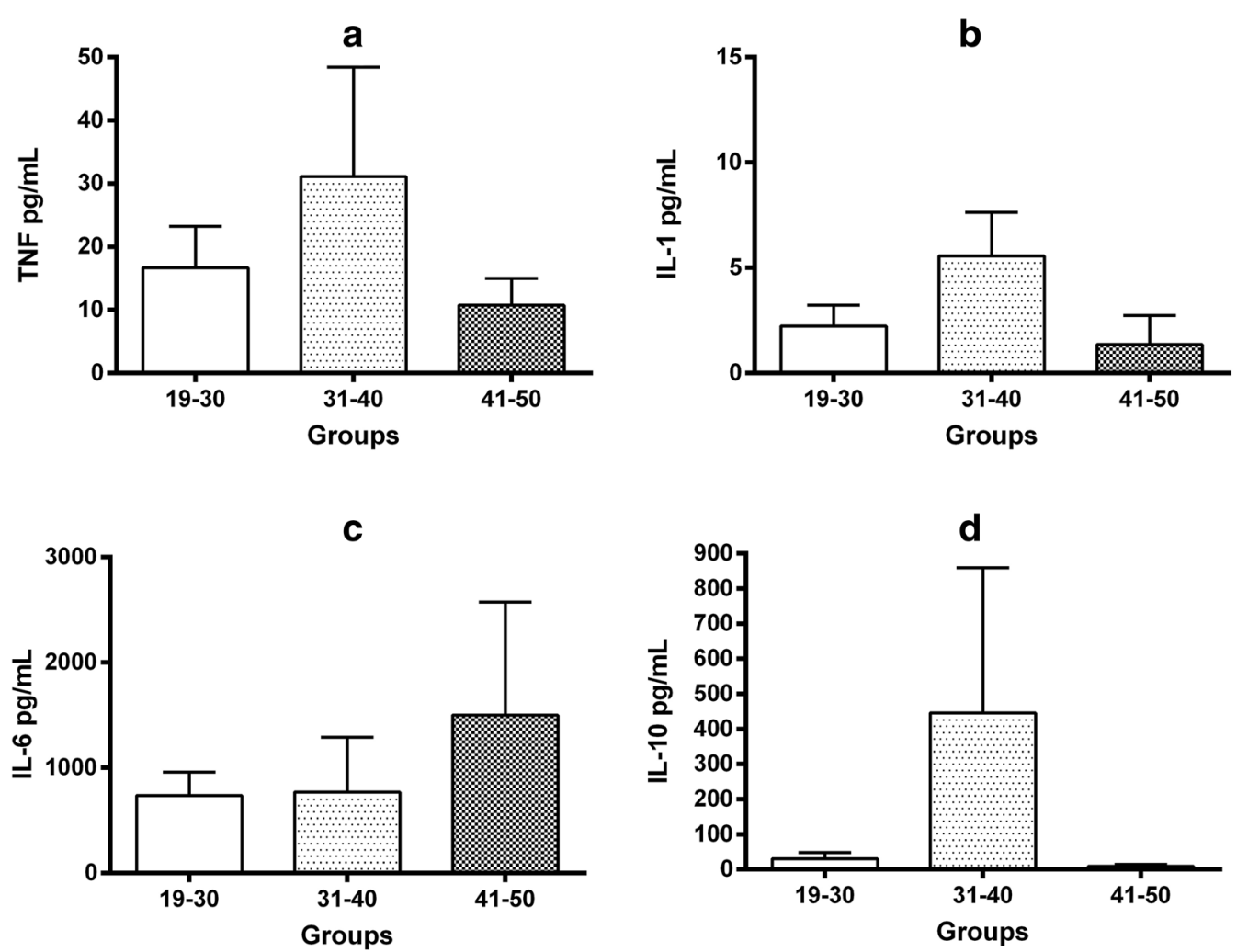

Fig. 1 Cytokine levels in FFP. TNF, IL-1ß, IL-10 and IL-6 ELISA measurements in FFP are represented in panels a-d respectively. Bars represent means and whiskers denote standard error. Measured were made for duplicate and the analyses between groups was made using a Kruskal-Wallis test with multiple comparison post-test by Dunn. Analyses were 2 -tailed and a $P<0.05$ value was used for significance

exposure to $20 \%$ FFP $(19-30$ years $=16.1 \pm 6.7 \mathrm{pg} /$ $\mathrm{mL}, \quad 31-40$ years $=27.1 \pm 11.8 \mathrm{pg} / \mathrm{mL}$, 41-50 years $=17.3 \pm 9.4 \mathrm{pg} / \mathrm{mL}$ ) (Fig. 2, panel g). In contrast, stimulation with LPS significantly increased IL-10 levels $(80.4 \pm 4.0 \mathrm{pg} / \mathrm{mL})$, to a much greater extent than observed in the LPS plus $20 \%$ FFP co-stimulation (19-30 years $=26.8 \pm 6.1 \mathrm{pg} /$ $\mathrm{mL}, \quad 31-40$ years $=23.2 \pm 6.6 \mathrm{pg} / \mathrm{mL}$, $41-50$ years $=29.1 \pm 10.4 \mathrm{pg} / \mathrm{mL} ; \mathrm{P}<0.001$ for all comparisons) (Fig. 2, panel h).

\section{U937 cells stimulated with $40 \%$ fresh frozen plasma}

In U937 cells cultured with RPMI, $25.9 \pm 2.0 \mathrm{pg} / \mathrm{mL}$ of TNF were measured, and stimulation with $40 \%$ FFP modified its levels to $74.1 \pm 36.1 \mathrm{pg} / \mathrm{mL}$ in $19-30$ years, $60.9 \pm 2.1 \mathrm{pg} / \mathrm{mL}$ in $31-40$ years, and $58.7 \pm 15.9 \mathrm{pg} /$ $\mathrm{mL}$ in $41-50$ years (Fig. 3, panel a). Co-stimulation with $40 \%$ FFP plus LPS was no associated with important changes in TNF levels $(19-30$ years $=50.9 \pm 13.2 \mathrm{pg} /$ $\mathrm{mL}, \quad 31-40$ years $=47.7 \pm 14.3 \mathrm{pg} / \mathrm{mL}$, and $41-50$ years $=246.7 \pm 208.0 \mathrm{pg} / \mathrm{mL}$ (Fig. 3, panel b). Concerning to IL-1 $\beta$, from a basal production of $0.9 \pm 0.9 \mathrm{pg} /$ $\mathrm{mL}$, stimulation with $40 \%$ FFP induced significant increased levels in groups 19-30 years $=29.9 \pm 8.4 \mathrm{pg} /$
$\mathrm{mL}$, and $41-50=64.0 \pm 39.1 \mathrm{pg} / \mathrm{mL}$, in the oldest group levels were higher than 31-40 years group $(22.1 \pm 6.0 \mathrm{pg} / \mathrm{mL} ; \mathrm{P}<0.05)$ (Fig. 3, panel c). Although stimulation with LPS alone increased IL-1 $\beta$ levels to $14.0 \pm 0.7 \mathrm{pg} / \mathrm{mL}$, co-stimulation with LPS plus $40 \%$ FFP no longer modified them $(19-30$ years $=16.5 \pm 6.5$, $31-40$ years $=14.6 \pm 2.4,41-50$ years $=25.3 \pm 11.5 \mathrm{pg} /$ $\mathrm{mL}$ ) (Fig. 3, panel d).

In unstimulated cultures, IL-6 level was $18.4 \pm 1.8 \mathrm{pg} /$ $\mathrm{mL}$, and exposure to $40 \%$ FFP resulted in $17.91 \pm 9.4$, $10.0 \pm 7.5$ and $75.3 \pm 75.3 \mathrm{pg} / \mathrm{mL}$ when co-cultured with FFP of $19-30,31-40$, and $41-50$ years, respectively (Fig. 3, panel e). LPS stimulation increased IL-6 levels $(126.5 \pm 8.0 \mathrm{pg} / \mathrm{mL}, \mathrm{P}<0.001$ in all comparisons with co-stimulated groups), while co-stimulation did not modify those levels observed with FFP alone $(19-30$ years $=8.8 \pm 3.3,31-40$ years $=5.0 \pm 3.1$, and $41-50$ years $=41.2 \pm 36.5 \mathrm{pg} / \mathrm{mL}$ ) (Fig. 3, panel f). Similar IL-10 levels were found in cells cultured with RPMI $(20.9 \pm 1.2 \mathrm{pg} / \mathrm{mL})$ and in cells stimulated with $40 \%$ FFP $(19-30$ years $=11.3 \pm 4.8 \mathrm{pg} /$ $\mathrm{mL}, \quad 31-40$ years $=25.0 \pm 8.1 \mathrm{pg} / \mathrm{mL}$, $41-50$ years $=25.9 \pm 10.4 \mathrm{pg} / \mathrm{mL}$ ) (Fig. 3, panel g). LPS stimulation alone increased IL-10 levels to 

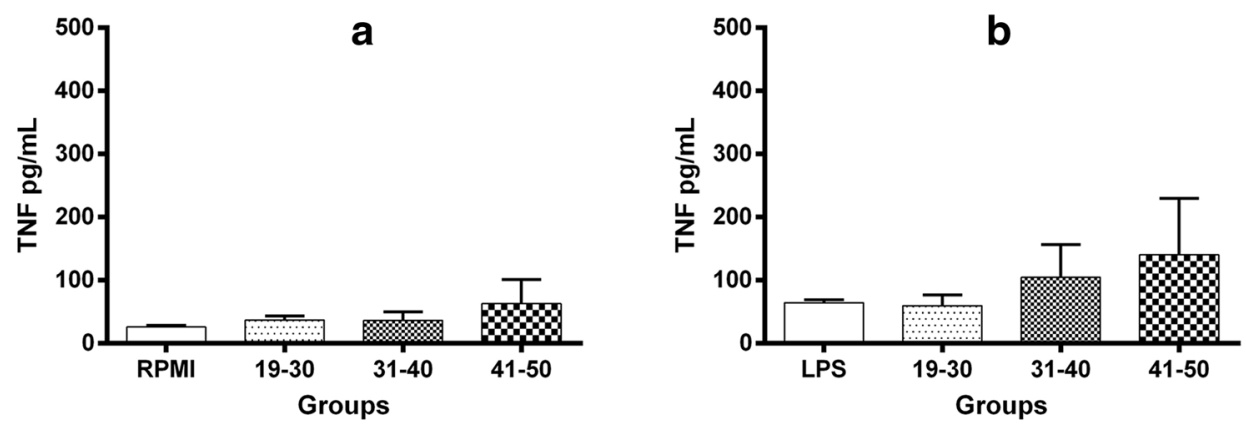

C

d
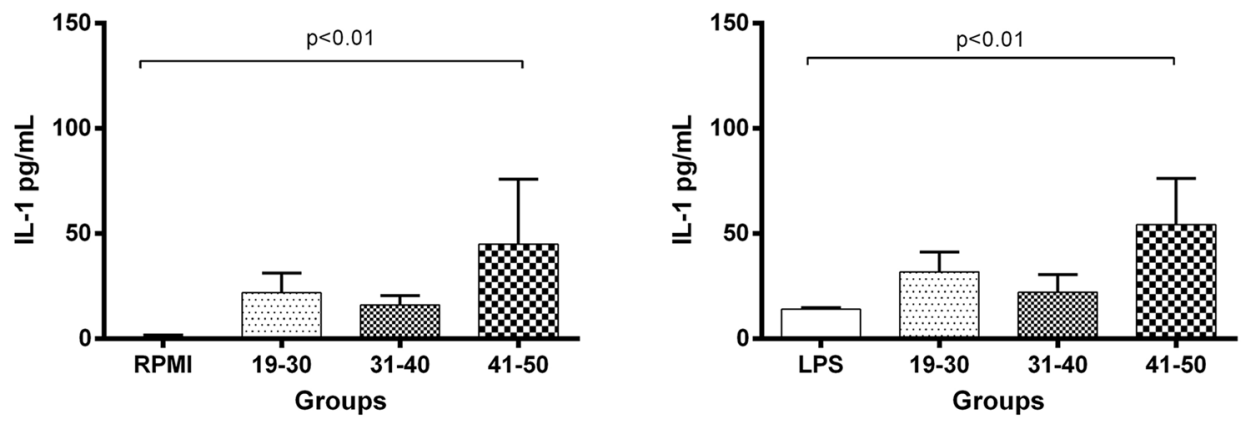

e
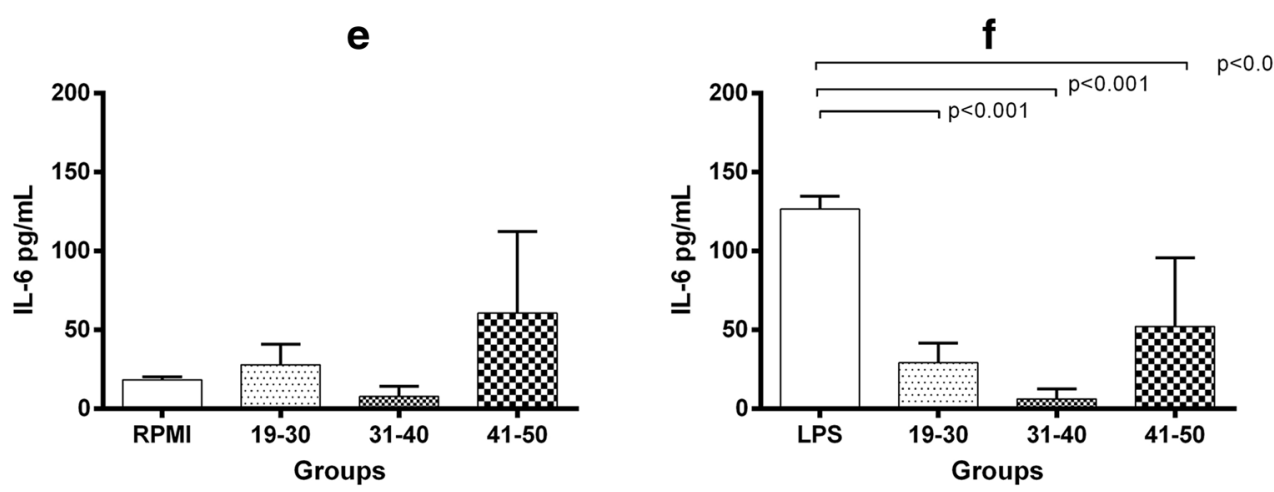

g
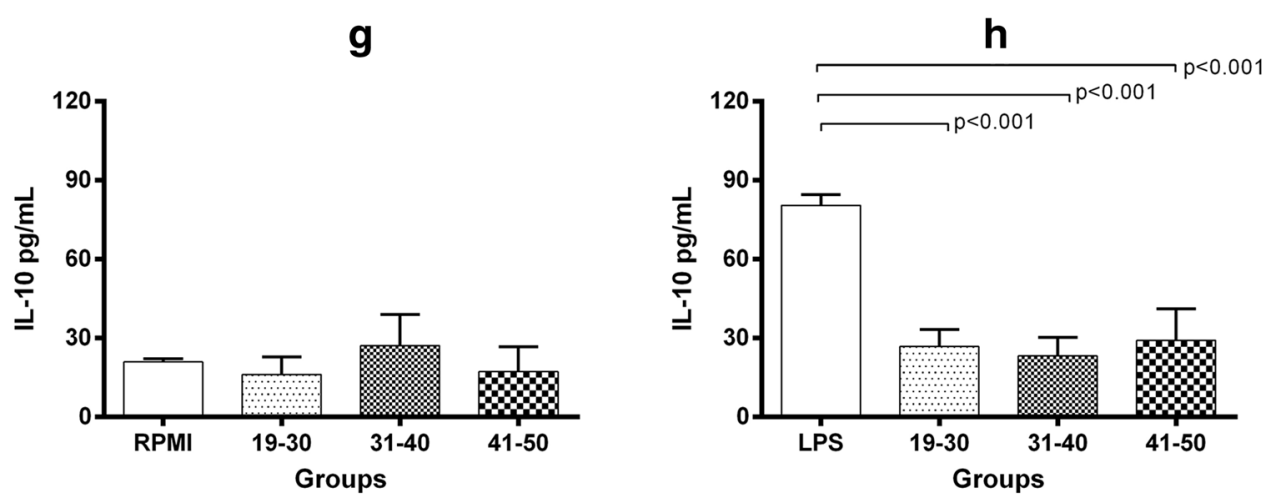

Fig. 2 Cytokine levels in U937 cell cultures with 20\% FFP.TNF, IL-1ß, IL-10 and IL-6 were measured by ELISA in supernatants from cultured cells stimulated during $24 \mathrm{~h}$ solely with 20\% FFP (panels $\mathbf{a}, \mathbf{c}, \mathbf{e}, \mathbf{g}$ ) or 20\% FFP plus LPS (panels $\mathbf{b}, \mathbf{d}, \mathbf{f}, \mathbf{h}$ ). Assays were performed in triplicate. Bars represent means and whiskers denote standard error. Statistical analyses were performed using the Kruskal-Wallis test with multiple comparison post-test by Dunn. Analyses were 2 -tailed, and a $P<0.05$ value was used for significance 

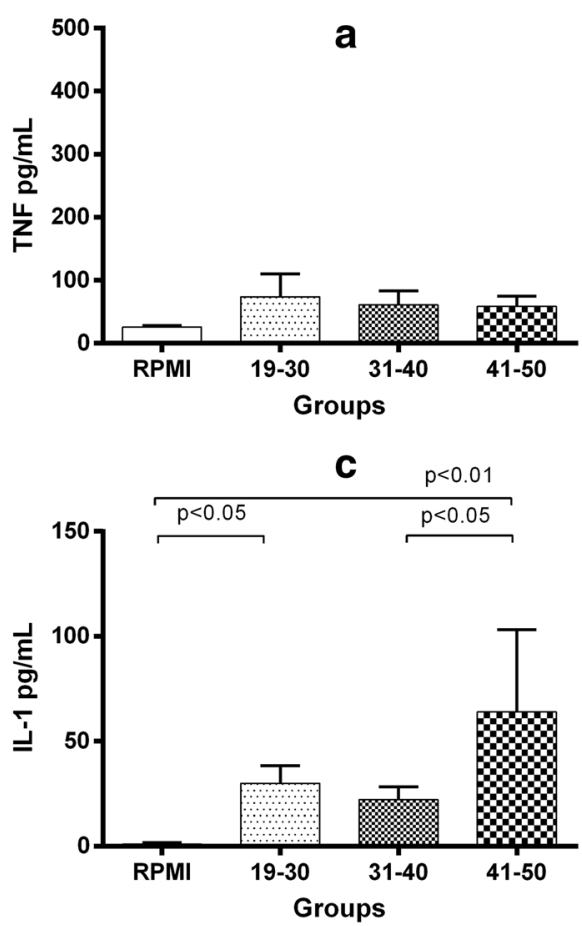

e

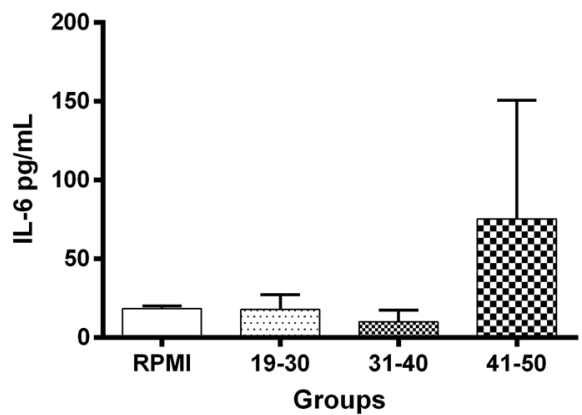

g

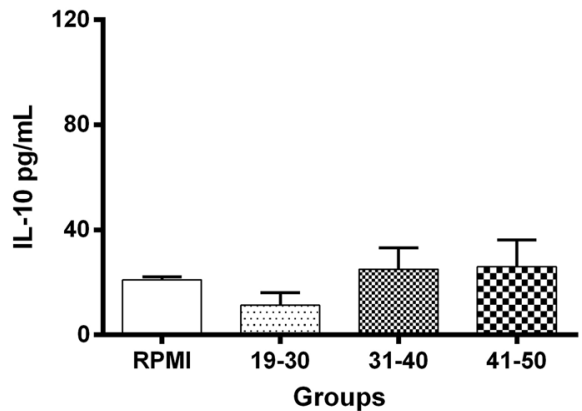

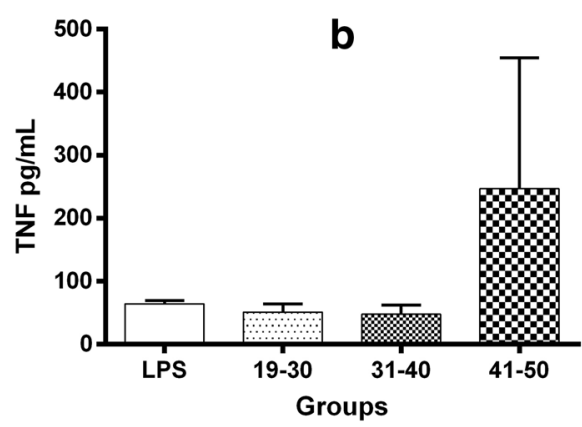

d
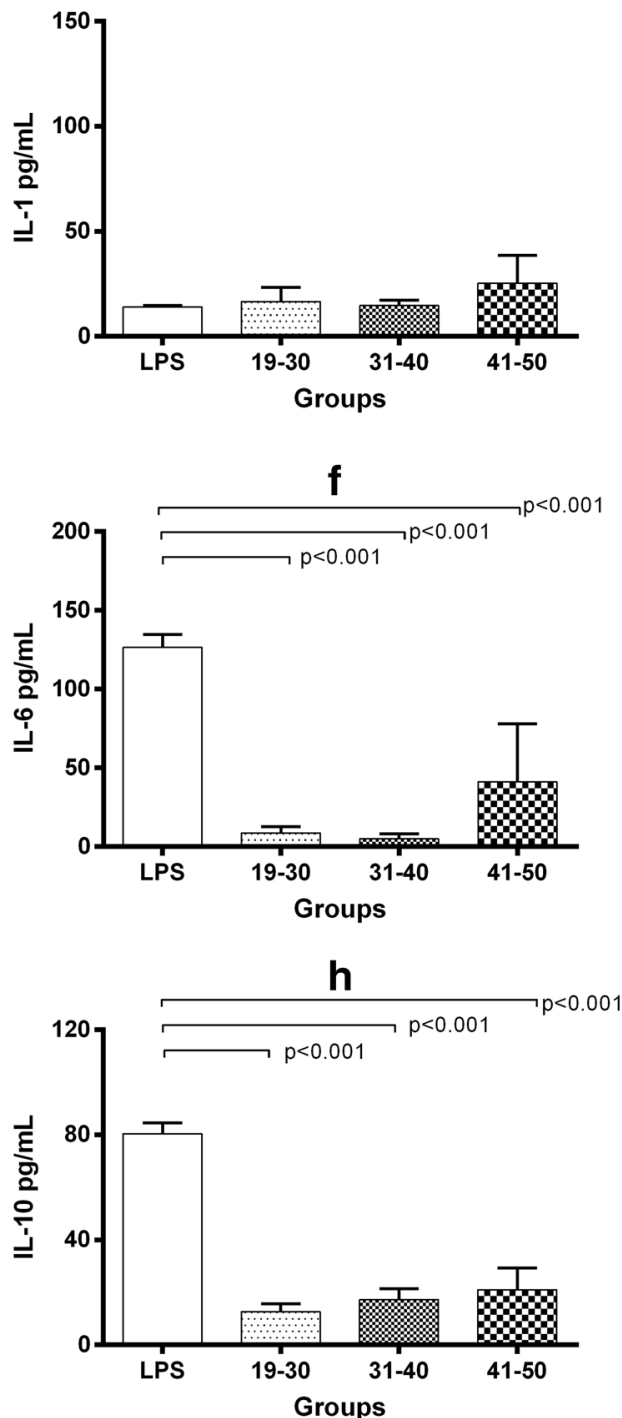

Fig. 3 Cytokine levels in U937 cell cultures with 40\% FFP. TNF, IL-1ß, IL-10 and IL-6 were measured by ELISA in supernatants from cultured cells stimulated during $24 \mathrm{~h}$ solely with 40\% FFP (panels $\mathbf{a}, \mathbf{c}, \mathbf{e}, \mathbf{g}$ ) or 40\% FFP plus LPS (panels $\mathbf{b}, \mathbf{d}, \mathbf{f}, \mathbf{h}$ ). Assays were performed in triplicate. Bars represent means and whiskers denote standard error. Statistical analyses were performed using the Kruskal-Wallis test with multiple comparison post-test by Dunn. Analyses were 2 -tailed, and a $\mathrm{P}<0.05$ value was used for significance 
$80.4 \pm 4.0 \mathrm{pg} / \mathrm{mL} \mathrm{P}<0.001$ for all comparisons), while co-stimulation with $40 \%$ FFP was associated to diminished IL-10 levels (19-30 years $=12.6 \pm 3.0 \mathrm{pg} /$ $\mathrm{mL}, \quad 31-40$ years $=17.2 \pm 5.2 \mathrm{pg} / \mathrm{mL}$, $41-50$ years $=21.0 \pm 8.3 \mathrm{pg} / \mathrm{mL})($ Fig. 3, panel h).

\section{Gene expression of activation molecules in U937 cells}

The relative expression of molecules related to activation in U937 cells was normalized according to the levels of expression found in unstimulated U937 cells (RPMI). Exposure to 20\% FFP of 19-30 years increased the expression of $C D 11 B$ to $295.0 \pm 270.0$, whereas this increased to $10.3 \pm 5.2$ and $85.7 \pm 80.1(\mathrm{P}<0.05)$ when exposed to FFP of $31-40$ and $41-50$ years, respectively (Fig. 4, panel a). Stimulation with LPS induced an increase in the expression of CD11B (86.6 \pm 51.3$)$, while the co-stimulation with LPS plus $20 \%$ FFP of individuals of $19-30,31-40$ and $41-50$ years resulted
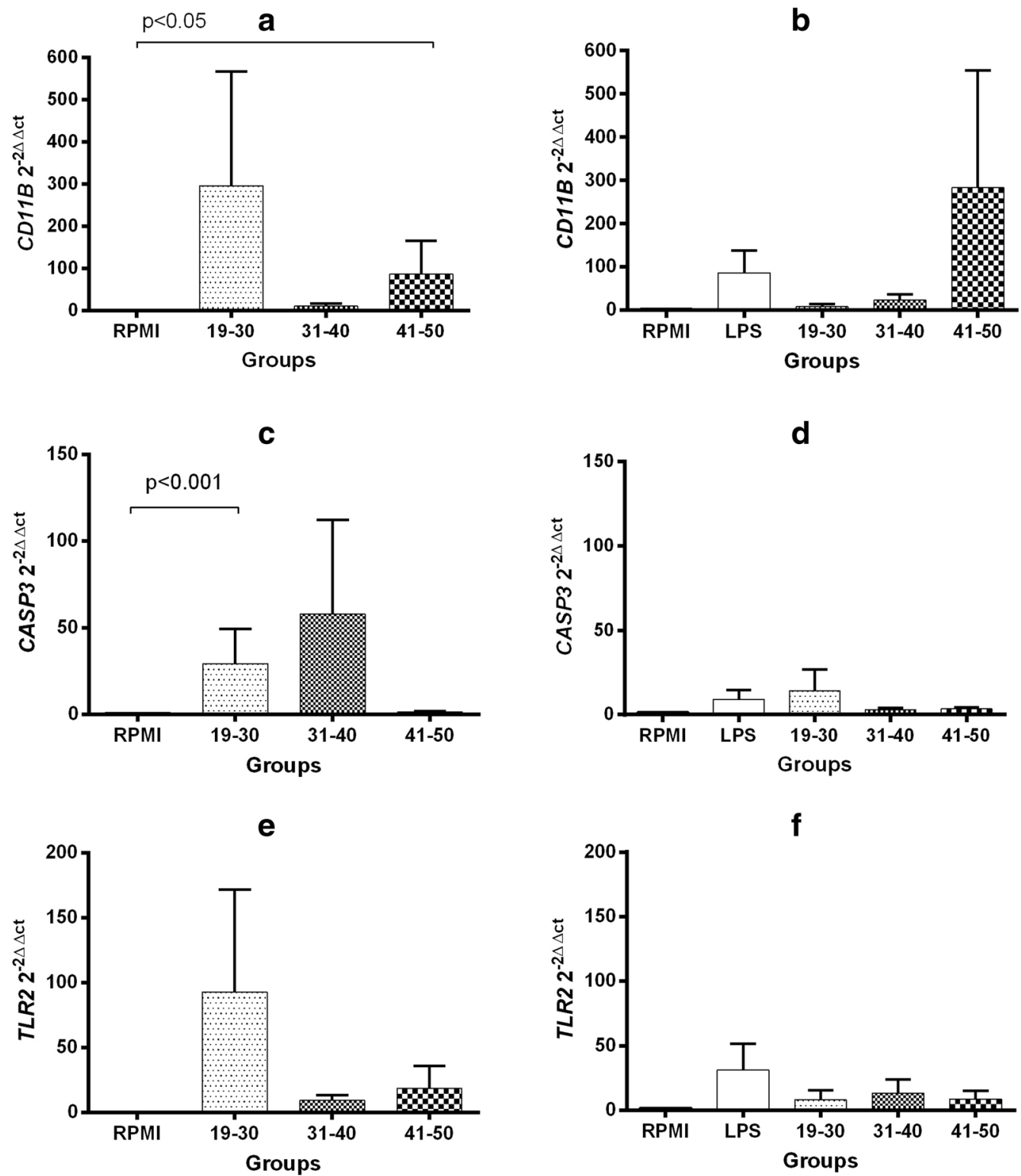

Fig. 4 mRNA levels of activation-related molecules in U937 cells with 20\% FFP. CD11b, Casp3 and TLR2 RT-qPCR measurements in cells stimulated $24 \mathrm{~h}$ solely with 20\% FFP (panels a, c, e) or 20\% FFP plus LPS (panels b, d, f). Bars represent means and whiskers denote standard error (mRNA levels according to the $2^{-\Delta \Lambda C T}$ method). Assays were performed in triplicate. Statistical analyses were performed using the Kruskal-Wallis test with multiple comparison post-test by Dunn. Analyses were 2 -tailed, and a $\mathrm{P}<0.05$ value was used for significance 
in $8.0 \pm 5.9,23.2 \pm 13.4$ and $283.2 \pm 271.4$ increased expression, respectively (Fig. 4, panel b). Exposure to $20 \%$ FFP of 19-30 years increased the expression of CASP3 to $29.3 \pm 19.9(\mathrm{P}<0.001)$, whereas this increased to $58.0 \pm 54.1,1.3 \pm 0.4$ when exposed to FFP of $31-40$ and 41-50 years, respectively (Fig. 4, panel c). Although stimulation with LPS increased the expression of CASP3 (9.2 \pm 5.3 ), co-stimulation with $20 \%$ FFP did not significantly modify this expression (19-30 years $=14.2 \pm 12.6$, $31-40$ years $=2.9 \pm 1.0,41-50$ years $=3.5 \pm 0.9$ ) (Fig. 4, panel d). The expression of TLR2 increased in the presence of $20 \%$ FFP (19-30 years $=92.7 \pm 78.7$, $31-40$ years $=9.1 \pm 3.8,41-50$ years $=31.3 \pm 20.3$ ) (Fig. 4, panel e). However, while stimulation with LPS induced an increase in TLR2 expression (31.3 \pm 20.3$)$, co-stimulation with LPS and 20\% FFP was not associated with significant changes in TLR2 expression $(19-30$ years $=8 \pm 7.5,31-40$ years $=13.3 \pm 9.7$, $41-50$ years $=8.7 \pm 6.3)$ (Fig. 4 , panel $\mathrm{f}$ ).

As can be observed in Fig. 5 (panels a-f), the relative expression of any of the activation-related molecules was not significantly modified with exposure to $40 \%$ FFP of any of the age groups, either alone or in co-stimulation with LPS.

\section{Phagocytosis assays}

Finally, total phagocytosis in U937 cells cultured with RPMI was used as $100 \%$ reference. The addition of $20 \%$ FFP was associated with a decrease in phagocytosis, although only the FFP of 31-40 years reached significance $(19-30$ years $=77 \% \pm 16 \%, 31-40$ years $=52.8 \% \pm 12.6 \%$, $41-50$ years $=69.9 \% \pm 25.5 \%$ ) (Fig. 6, panel a). When phagocytic activity was evaluated in the presence of $40 \%$ FFP, a significant reduction was observed only in the $19-30$ years $(68.8 \% \pm 18.8 \%$; $\mathrm{P}<0.05)$, and $31-40$ years $(62.1 \% \pm 13.7 \% ; \mathrm{P}<0.05)$ groups (Fig. 6, panel b).

\section{Discussion}

This study assessed the effect of FFP from healthy donors grouped by age, on the in vitro activation of human cells from monocytic lineage. Here, we found that FFP exposure was associated with modifications in the production of inflammatory cytokines, the expression of activationrelated genes, and in total phagocytosis. In addition, this study highlighted that age of blood donors as well as the type of cytokines present in plasma are relevant for both the magnitude and direction of inflammatory cell regulation.

Administration of FFP and other blood derivatives has been a cornerstone of the practice of medicine, although the development of serious side effects remains a major concern especially in critically ill patients $[19,21]$. The presence of soluble mediators in blood derivatives may be causative of several of these side effects since they have the potential to modulate immune cells in the host [14]. In line with this, FFP exposure was associated with increased production of TNF and IL-1 $\beta$, while precluded LPS-related activation and decreased phagocytosis in U937 cells. Therefore, our results suggest that FFP may act as an external source of cytokines, which in turn could induce transfusion-related, immune-mediated adverse events.

An important novelty of this study is the finding that the age of blood donors may have a role in the modulation of cell activation after FFP exposure. Indeed, a differential expression of cytokines in each age stratum might be responsible for individual cell responses in the host. An equalized TNF/IL-10 concentration in the presence of high IL-6 levels, as seen in FFP from 41 to 50 group, was associated with U937 cells activation and increased expression of $C D 11 B$ and $T L R 2$, even though a decreased phagocytic activity was observed. In contrast, higher IL-10 levels than TNF levels, as observed in FFP from 19 to 30 and 31 to 40 groups, was associated with decreased $C D 11 B$ and TLR2 expression but increased $C A S P 3$ expression. Notably, the highest CASP3 expression was found to be associated with decreased phagocytic activity. Despite endogenous TNF or IL-10 cannot explain the reduction in CASP3 expression, the presence of high levels of IL-6 in FFP from individuals aged 41-50 could explain these results, at least partially. Indeed, IL-6 may suppress apoptosis through PI3-Kinase/Akt activation via the phosphorylation of BCL-2 family member BAD. Phosphorylated BAD is associated with 14-3-3 proteins which sequester BAD from BCL- $\mathrm{X}_{\mathrm{L}}$, thereby promoting cell survival [29]. In addition, IL-6 may prevent CASP3 overexpression by inhibiting IP3R-dependent $\mathrm{Ca}^{2+}$ release-induced apoptosis [30]. Accordingly, FFP from donors aged 19-31 showed the lowest levels of IL-6 and these samples were responsible for the highest expression of $C A S P 3$ as an apoptosis marker. This is further supported by the demonstration that IL- 6 blockade may reduce tumor cell viability while promotes apoptosis [31]. Despite that stimulation with LPS significantly increased both cytokine production and expression of activation-related molecules, cells co-cultured with FFP presented only a slight increment in the TNF production as well as a decreased expression of activation molecules. Previously, Schneider and colleagues have shown that addition of a variety of blood derivatives such as PRBC or FFP significantly suppresses TNF production, especially in monocytes stimulated with LPS plus FFP [12]. The mechanisms underlying the inhibitory response associated with blood derivatives remain obscure, but a plausible explanation seems to involve the presence of soluble molecules in FFP. In fact, IL-1 receptor antagonist 

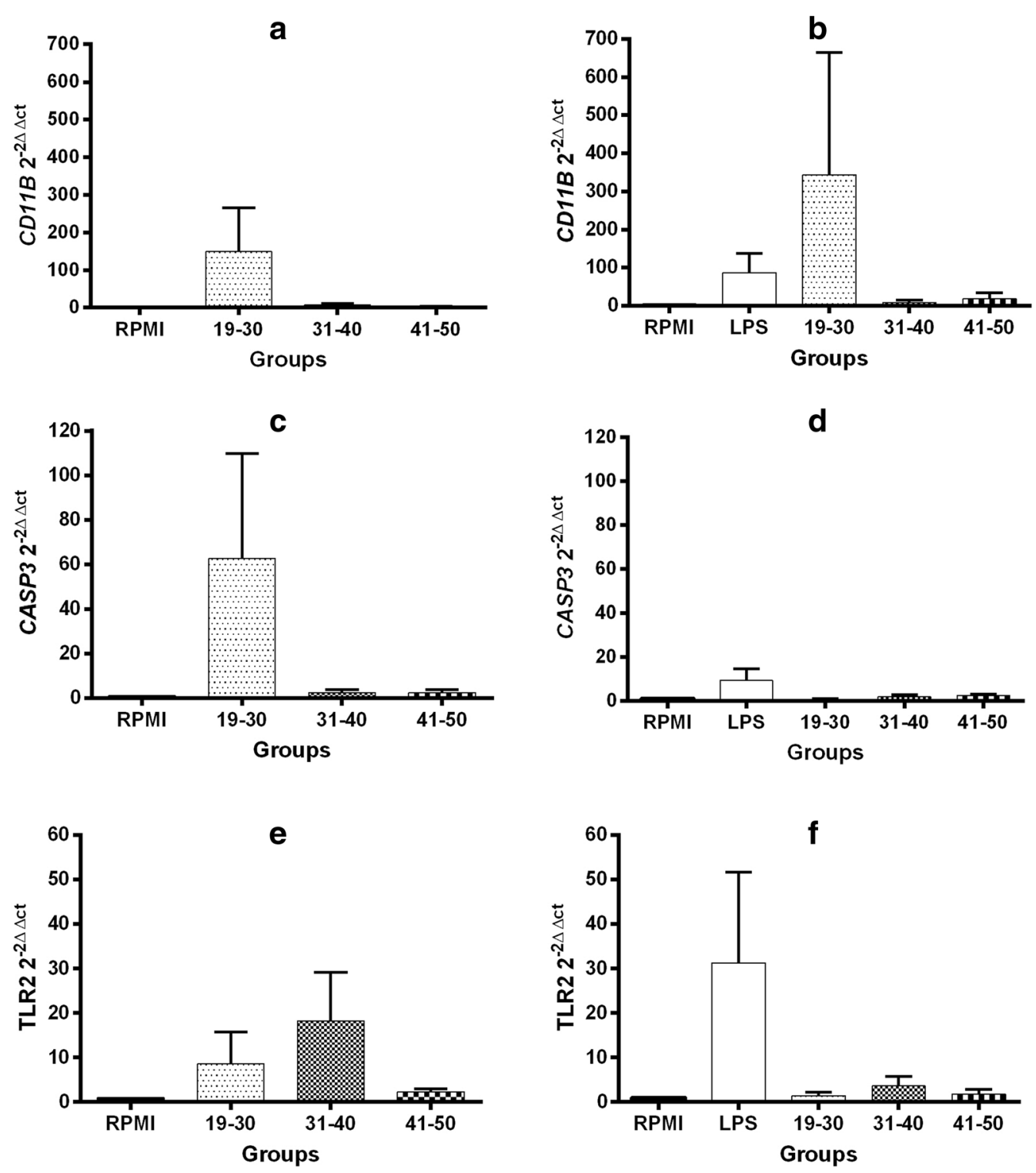

Fig. 5 mRNA levels of activation-related molecules in U937 cells with 40\% FFP. CD1 1 b, Casp3 and TLR2 RT-qPCR measurements in cells stimulated $24 \mathrm{~h}$ solely with $40 \%$ FFP (panels a, c, e) or 40\% FFP plus LPS (panels b, d, f). Bars represent means and whiskers denote standard error (mRNA levels according to the $2^{-\triangle \Delta C T}$ method). Assays were performed in triplicate. Statistical analyses were performed using the Kruskal-Wallis test with multiple comparison post-test by Dunn. Analyses were 2-tailed, and a $\mathrm{P}<0.05$ value was used for significance

(IL-1RA) as well as the soluble TNF receptors (sTNFR) I and II are elevated in the serum of healthy individuals [32, 33]. These soluble receptors are known to bind directly to the free cytokine, thereby competing with membrane-bound receptors. The final effect of these soluble receptors may be the interference in cellular actions, altering the production of other cytokines. Alternatively, serum IgM and IgG anti-LPS antibodies have already been demonstrated in Balb/c mice treated with LPS, and anti-LPS antibodies with antibacterial activity were recently described in humans $[34,35]$.
Finally, even though FFP and other blood derivatives may approve the scrutiny of blood banks for infectious agents, they can bring cytokines and other soluble mediators reflecting inflammatory subclinical events occurring in otherwise healthy blood donors. Moreover, the normal physiological response that underlies any individual is changing during the course of life and it may be reflected in the cytokine levels according to the age of blood donors [14, 24, 25, 36].

We are aware that our study has several limitations. Firstly, we focused this work to assess several cytokines 


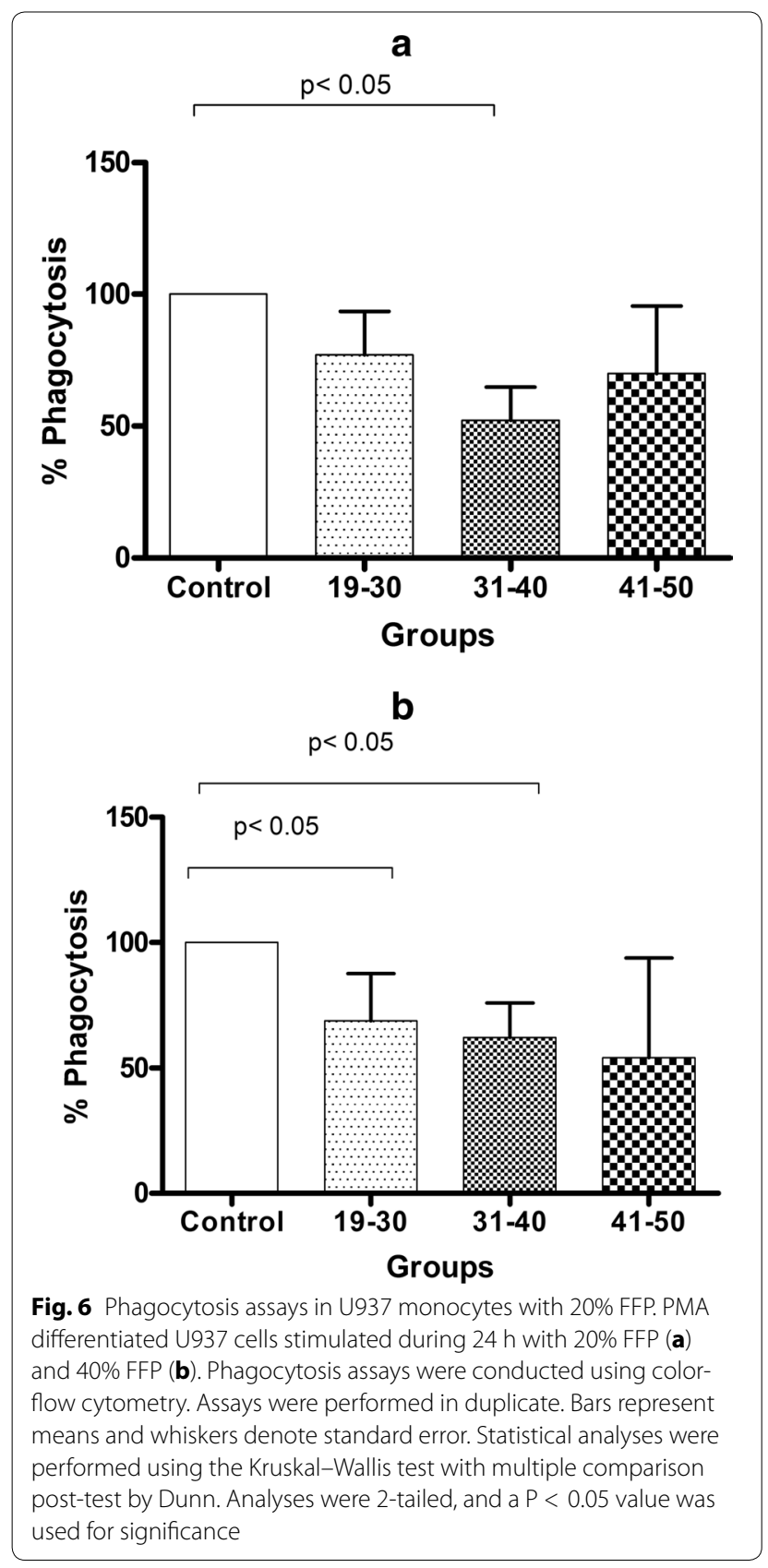

mainly found in early inflammatory responses, and the role that adaptive immune cytokines may play in monocyte activation remains to be elucidated. Secondly, we were unable to specifically inhibit each cytokine with specific anti-cytokine monoclonal antibodies due to technical difficulties, thus confirmation of a direct cause-effect relationship is still lacking. The role that age-related sex hormones conveyed in FFP may play in cell activation was not assessed. Finally, although FFP from a blood bank were used in this study, we must consider that other factors associated with donors' lifestyle could influence the large differences observed in response to monocyte stimulation.

In conclusion, this study supports the notion that soluble mediators in FFP may modulate the activation and overall functioning of monocytes. In addition, our results suggest that these effects are related to the age of blood donors.

\begin{abstract}
Abbreviations
CASP: caspase; CD: cluster of differentiation molecules; CDNA: complementary DNA; ELISA: enzyme-linked immunosorbent assay; FFP: fresh frozen plasma; HEPES: (4-(2-hydroxyethyl)-1-piperazineethanesulfonic acid) buffer; IL-: interleukin; IP3: inositol triphosphate; LPS: lipopolysaccharide; PCR: polymerase chain reaction; PRBC: packed red blood cells; QPCR: real-time polymerase chain reaction; RNA: ribonucleic acid; RPMI: Roswell Park Memorial Institute medium; TLR: toll-like receptor; TNF: tumor necrosis factor; TRALI: transfusionrelated acute lung injury; UPL: Universal Probe Library Roche.
\end{abstract}

\section{Authors' contributions}

MP, cell cultures, apoptosis assays, molecular analysis and immunoassays; FSM, molecular analysis; LMAG, study design and draft the manuscript; AG, immunoassays; AP; cell cultures and apoptosis assays; FM, cell cultures and color-flow cytometry; AMM and AS, plasma processing; RB and LP, data analysis; LAJS and RMV, protocol design, technical advice, analysis of results and drafting the manuscript. All authors read and approved the final manuscript.

\section{Author details}

${ }^{1}$ Doctorado en Ciencias Quimicobiológicas, Escuela Nacional de Ciencias Biológicas, Instituto Politécnico Nacional, Mexico City, Mexico. ${ }^{2}$ Department of Immunology, Instituto Nacional de Cardiología Ignacio Chávez, Juan Badiano No1, Col Sección XVI, 14080 Mexico City, Mexico. ${ }^{3}$ Department of Health Care, Universidad Autónoma Metropolitana-Xochimilco, Mexico City, Mexico. ${ }^{4}$ Department of Physiology, Instituto Nacional de Cardiología Ignacio Chávez, Mexico City, Mexico. ${ }^{5}$ Blood Bank, Instituto Nacional de Cardiología Ignacio Chávez, Mexico City, Mexico. ${ }^{6}$ Neuropsicoimmunology Laboratory, Instituto Nacional de Psiquiatría Ramón de la Fuente, Mexico City, Mexico. ${ }^{7}$ Department of Immunology, Escuela Nacional de Ciencias Biológicas, Instituto Politecnico Nacional, Prolongación de Carpio y Plan de Ayala s/n, Santo Tomas, 11340 Mexico City, Mexico.

\section{Acknowledgements}

Not applicable.

\section{Competing interests}

The authors declared that they have no competing interests.

\section{Availability of data and materials}

The data set supporting our results are included within the article.

\section{Consent for publication}

\section{Not applicable.}

\section{Ethical approval and consent to participate}

This study was approved by the local ethics committee under reference number 13-801. Informed consent is not applicable for this publication since we only used leftovers from plasma processing at the Blood bank.

\section{Funding}

Not applicable.

\section{Publisher's Note}

Springer Nature remains neutral with regard to jurisdictional claims in published maps and institutional affiliations.

Received: 24 January 2017 Accepted: 4 December 2017

Published online: 21 December 2017 


\section{References}

1. Spence RK. Clinical use of plasma and plasma fractions. Best Pract Res Clin Haematol. 2006;19:83-96.

2. Nascimento B, Callum J, Rubenfeld G, Neto JB, Lin Y, Rizoli S. Clinical review: fresh frozen plasma in massive bleedings - more questions than answers. Crit Care. 2010;14:202.

3. Pandey S, Vyas GN. Adverse effects of plasma transfusion. Transfusion. 2012;52(Suppl 1):65S-79S

4. Rao MP, Boralessa H, Morgan C, Soni N, Goldhill DR, Brett SJ, et al. Blood component use in critically ill patients. Anaesthesia. 2002;57:530-4.

5. Lauzier F, Cook D, Griffith L, Upton J, Crowther M. Fresh frozen plasma transfusion in critically ill patients. Crit Care Med. 2007;35:1655-9.

6. Benson AB. Pulmonary complications of transfused blood components. Crit Care Nurs Clin N Am. 2012;24:403-18.

7. Parreiras VC, Rocha Ide S, Martins AS, Meira EB, Taniguchi FP. Influence of fresh frozen plasma as a trigger factor for kidney dysfunction in cardiovascular surgery. Rev Bras Cir Cardiovasc. 2012;27:405-10.

8. Görlinger K, Saner FH. Prophylactic plasma and platelet transfusion in the critically III patient: just useless and expensive or even harmful? BMC Anesthesiol. 2015:15:86.

9. Sarani B, Dunkman WJ, Dean L, Sonnad S, Rohrbach Jl, Gracias VH. Trans fusion of fresh frozen plasma in critically ill surgical patients is associated with an increased risk of infection. Crit Care Med. 2008:36:1114-8.

10. Sadjadi J, Cureton EL, Twomey P, Victorino GP. Transfusion, not just injury severity, leads to posttrauma infection: a matched cohort study. Am Surg. 2009;75:307-12.

11. Muszynski J, Nateri J, Nicol K, Greathouse K, Hanson L, Hall M. Immunosuppressive effects of red blood cells on monocytes are related to both storage time and storage solution. Transfusion. 2012:52:794-802.

12. Schneider SO, Rensing H, Gräber S, Kreuer S, Kleinschmidt S, Kreimeier $S$, et al. Impact of platelets and fresh frozen plasma in contrast to red cell concentrate on unstimulated and stimulated cytokine release in an in vitro model of transfusion. Scand J Immunol. 2009:70:101-5.

13. Muszynski JA, Bale J, Nateri J, Nicol K, Wang Y, Wright V, et al. Supernatants from stored red blood cell (RBC) units, but not RBC-derived microvesicles, suppress monocyte function in vitro. Transfusion. 2015;55:1937-45.

14. Theusinger OM, Baulig W, Seifert B, Emmert MY, Spahn DR, Asmis LM. Relative concentrations of haemostatic factors and cytokines in solvent/detergent-treated and fresh-frozen plasma. Br J Anaesth. 2011;106:505-11.

15. Schulte W, Bernhagen J, Bucala R. Cytokines in sepsis: Potent immunoregulators and potential therapeutic targets - an updated view. Mediat Inflamm. 2013;2013:165974. https://doi.org/10.1155/2013/165974.

16. Abrahamsohn IA. Cytokines in innate and acquired immunity to Trypanosoma cruzi infection. Braz J Med Biol Res. 1998;31:117-21.

17. Cata JP, Wang H, Gottumukkala V, Reuben J, Sessler DI. Inflammatory response, immunosuppression, and cancer recurrence after perioperative blood transfusions. Br J Anaesth. 2013;110:690-701.

18. Bux J, Sachs UJH. The pathogenesis of transfusion-related acute lung injury (TRALI). Br J Haematol. 2007;136:788-99.

19. Williams CMM, Rahman S, Hubeau C, Ma H-L. Cytokine pathways in allergic disease. Toxicol Pathol. 2012;40:205-15

20. Davenport RD. Cytokines as intercellular signals in hemolytic transfusion reactions. Biol Signals. 1996;5:240-5.

21. Roubinian NH, Looney MR, Kor DJ, Lowell CA, Gajic O, Hubmayr RD, et al. Cytokines and clinical predictors in distinguishing pulmonary transfusion reactions. Transfusion. 2015:55:1838-46.
22. Klabusay M, Kohutova V, Coupek P, Nenickova M, Tesarova E. Simultaneous analysis of cytokines and co-stimulatory molecules concentrations by ELISA technique and of probabilities of measurable concentrations of interleukins IL-2, IL-4, IL-5, IL-6, CXCL8 (IL-8), IL-10, IL-13 occurring in plasma of healthy blood don. Mediat Inflamm. 2006;2006:1-7.

23. Urner M, Herrmann IK, Buddeberg F, Schuppli C, Z'graggen BR, Hasler M, et al. Effects of blood products on inflammatory response in endothelial cells in vitro. PLoS ONE. 2012;7:e33403.

24. Kleiner G, Marcuzzi A, Zanin V, Monasta L, Zauli G. Cytokine levels in the serum of healthy subjects. Mediat Inflamm. 2013;2013:434010. https:// doi.org/10.1155/2013/434010.

25. Kim H, Kim H-S, Youn J-C, Shin E-C, Park S. Serum cytokine profiles in healthy young and elderly population assessed using multiplexed beadbased immunoassays. J Transl Med. 2011;9:113.

26. Forsey RJ, Thompson JM, Ernerudh J, Hurst TL, Strindhall J, Johansson $B$, et al. Plasma cytokine profiles in elderly humans. Mech Ageing Dev. 2003;124:487-93.

27. Basu D, Kulkarni R. Overview of blood components and their preparation. Indian J Anaesth. 2014;58:529-37.

28. "Para la disposición de sangre humana y sus componentes con fines terapéuticos". Norma Oficial Mexicana NOM-253-SSA1-2012. Diario Oficial de la Federación. 2012.

29. Chen RH, Chang MC, Su YH, Tsai YT, Kuo ML. Interleukin-6 inhibits transforming growth factor-beta-induced apoptosis through the phosphatidylinositol 3-kinase/Akt and signal transducers and activators of transcription 3 pathways. J Biol Chem. 1999;274:23013-9.

30. Qiu AW, Yang Q, Yuan ST, Xie P, Liu QH. Interleukin-6 prevents NMDAinduced neuronal death via Gp130 signaling-dependent IP3R inhibition. Neuro Endocrinol Lett. 2013:34:529-38.

31. Wang ZY, Zhang JA, Wu XJ, Liang YF, Lu Y Bin, Gao YC, et al. IL-6 Inhibition Reduces STAT3 Activation and Enhances the Antitumor Effect of Carboplatin. Mediat Inflamm. 2016;2016:8026494. https://doi. org/10.1155/2016/8026494.

32. Nakai Y, Hamagaki S, Takagi $R$, Taniguchi A, Kurimoto F. Plasma concentrations of tumor necrosis factor-a (TNF-a) and soluble TNF receptors in patients with anorexia nervosa. J Clin Endocrinol Metab. 1999;84:1226-8.

33. Aukrust P, Frøland SS, Liabakk NB, Müller F, Nordøy I, Haug C, et al. Release of cytokines, soluble cytokine receptors, and interleukin-1 receptor antagonist after intravenous immunoglobulin administration in vivo. Blood. 1994:84:2136-43.

34. Márquez-Velasco R, Massó F, Hernández-Pando R, Montaño LF, Springall $R$, Amezcua-Guerra LM, et al. LPS pretreatment by the oral route protects against sepsis induced by cecal ligation and puncture. Regulation of proinflammatory response and lgM anti-LPS antibody production as associated mechanisms. Inflamm Res. 2007;56:385-90.

35. Schmiel DH, Moran EE, Keiser PB, Brandt BL, Zollinger WD. Importance of antibodies to lipopolysaccharide in natural and vaccine-induced serum bactericidal activity against Neisseria meningitidis group B. Infect Immun. 2011:79:4146-56.

36. Pak S, Holland N, Garnett EA, Mileti E, Mahadevan U, Beckert R, et al. Cytokine profiles in peripheral blood of children and adults with Crohn disease. J Pediatr Gastroenterol Nutr. 2012;54:769-75. 